\title{
Nucleus Reuniens Thalami Modulates Activity in Hippocampal Field CA1 through Excitatory and Inhibitory Mechanisms
}

\author{
M. J. Dolleman-Van der Weel, ${ }^{1,2}$ F. H. Lopes da Silva, ${ }^{2}$ and M. P. Witter ${ }^{1}$ \\ ${ }^{1}$ Graduate School for Neurosciences Amsterdam, Research Institute for Neurosciences, Faculty of Medicine, Department \\ of Anatomy and Embryology, Vrije Universiteit, 1081 BT Amsterdam, The Netherlands, and 2Institute of Neurobiology, \\ Faculty of Biology, University of Amsterdam, 1098 SM Amsterdam, The Netherlands
}

The nucleus reuniens thalami (RE) originates dense projections to CA1, forming asymmetrical synapses on spines (50\%) and dendrites (50\%). The hypothesis that RE input modulates transmission in CA1 through excitation of both pyramidal cells and interneurons was tested using electrophysiological methods in the anesthetized rat. The RE-CA1 afferents were selectively stimulated at their origin; evoked field potentials and unit activity were recorded in CA1. RE-evoked depth profiles showed a prominent negative deflection in the stratum lacunosummoleculare and a positive one in the stratum radiatum. The lacunosum-moleculare sink-radiatum source configuration is compatible with RE-elicited depolarization of apical dendrites of pyramidal cells. Despite a consistent and robust paired pulse facilitation of RE-evoked field potentials, population spikes in the stratum pyramidale were not detected at any tested condition. This indicates the inability of RE-CA1 input to discharge pyramidal cells. However, stimulation of RE-elicited spiking of extracellularly recorded units in strata oriens/alveus and distal radiatum, indicative of the activation of local interneurons. Thus, RE seems to modulate transmission in CA1 through a (subthreshold) depolarization of pyramidal cells and a suprathreshold excitation of putative inhibitory oriens/alveus and radiatum interneurons.

RE-evoked monosynaptic or disynaptic field potentials were associated with stimulation of rostral or caudal RE, respectively. Anatomically, a projection from caudal to rostral RE was demonstrated that can account for the disynaptic RE-CA1 input. Because caudal $R E$ receives input from the hippocampus via the subiculum, we propose the existence of a closed REhippocampal circuit that allows RE to modulate the activity in CA1, depending on hippocampal output.

Key words: rat; electrophysiology; neuroanatomical tracing; hippocampus; midline thalamus; limbic system; learning and memory
The involvement of thalamic midline nuclei in early stages of Alzheimer's disease (Braak and Braak, 1991, 1992) and in diencephalic amnesia (Rousseau, 1994) has recently drawn attention to the connectivity between the nucleus reuniens (RE) and structures of the medial temporal lobe (Herkenham, 1978; Wouterlood et al., 1990; Dolleman-Van der Weel and Witter, 1996). In this study we focused on the RE projection to hippocampal field CA1, a crucial structure for learning and memory processes (Squire, 1992).

A few investigators have studied the contribution of RE to hippocampal functioning. Vanderwolf et al. (1985) examined whether the medial thalamic nuclei, including RE, were involved in generating hippocampal atropine-resistant theta rhythm. Their extensive radiofrequency lesions, however, resulted in little or no effect on this type of rhythmical activity. Hirayasu and Wada (1992a,b) injected NMDA in the thalamic midline of the rat. When NMDA was administered to RE, it caused tonic and/or clonic generalized convulsions associated with temporal limbic EEG seizure discharge. They proposed that RE participates in the modulation of temporal limbic excitability and seizure development. Their findings suggest that RE influences the state of activity of the entorhinal-hippocampal circuit. Yet, basic electro-

Received Feb. 27, 1997; revised May 8, 1997; accepted May 8, 1997.

This work was supported by Neurowetenschappen Amsterdam Grant 90-20 from the Graduate School for Neurosciences Amsterdam. We thank Professor W. J. Wadman for his advice and A. J. A. Juta for helping with the calculation of the CSDs.

Correspondence should be addressed to Menno P. Witter, Department of Anatomy and Embryology, Faculty of Medicine, Vrije Universiteit, 7 van der Boechorststraat, 1081 BT Amsterdam, The Netherlands.

Copyright (C) 1997 Society for Neuroscience $\quad 0270-6474 / 97 / 175640-11 \$ 05.00 / 0$ physiological knowledge of RE is still lacking. A modulatory role for RE in normal functioning of the hippocampus has also been suggested based on anatomical data (Wouterlood et al., 1990; Dolleman-Van der Weel and Witter, 1996). Within field CA1, RE fibers terminate in a dense laminar plexus confined to the stratum lacunosum-moleculare (L-M) and form exclusively asymmetrical (i.e., excitatory) synapses on spines $(50 \%)$ and dendrites $(50 \%)$ (Wouterlood et al., 1990). These data indicate that RE fibers contact the spinous apical dendrites of pyramidal cells, as well as the largely aspinous dendrites of interneurons.

Based on the latter anatomical observations and the findings by Hirayasu and Wada (1992a,b), we proposed that RE modulates transmission in CA1 through activation of both pyramidal and nonpyramidal cells. This hypothesis was tested using electrophysiological methods to stimulate the RE-CA1 afferents selectively in anesthetized rats. RE-CA1 fibers course mainly within the inferior thalamic peduncle in a rostral direction, curve dorsally around the genu of the corpus callosum, and then run caudally via the cingulate bundle (in which many hippocampal afferents and efferents course) to enter CA1 (Wouterlood et al., 1990). Within their terminal field in stratum L-M, RE axons overlap with the perforant path fibers from the entorhinal cortex (Herkenham, 1978; Wouterlood et al., 1990; Dolleman-Van der Weel et al., 1994). Thus, stimulation of RE-CA1 fibers neither in the cingulate bundle nor within their terminal field in stratum L-M can provide the selectivity required. Therefore, the RE neurons had to be stimulated at their origin, and depth profiles of evoked field potentials and recordings of unit activity were performed in CA1. 
Our electrophysiological observations indicated the occurrence of monosynaptic and disynaptic RE-CA1 input. To substantiate a possible intrinsic RE connectivity, which is likely to be involved in disynaptically evoked CA1 responses, a neuroanatomical tracing method was used.

\section{MATERIALS AND METHODS}

We used 30 male Wistar rats (Harlan CPB, Zeist, The Netherlands), weighing 275-375 gm. Under halothane anesthesia the trachea was intubated. The animal was then placed in a stereotaxic apparatus and throughout the experiment artificially ventilated by a mixture of $\mathrm{O}_{2}$ and $\mathrm{N}_{2} \mathrm{O}$ with $1 \%$ halothane. Body temperature was maintained using a heating pad. The skull was exposed, and two burr holes were made on the left side of the brain to accommodate placement of stimulating and recording electrodes in $\mathrm{RE}$ and $\mathrm{CA} 1$, respectively. Because the most dorsal (i.e., septal) part of CA1 is less densely innervated by RE fibers than the ventral (temporal) part (Wouterlood et al., 1990), recordings were made approximately at the level of the splenium of the corpus callosum, or slightly more caudal (see Fig. $1 A, B$ ). Stereotaxic coordinates were derived from Paxinos and Watson (1986). They were zeroed at bregma (Br.), the midline of the sinus, and the cortical (dura) surface [stimulation electrode in $\mathrm{RE}$ at an angle of $15^{\circ}$ in the coronal plane: $\mathrm{Br}$., $-1.80 \mathrm{~mm}$; lateral $(\mathrm{L}), 2.0 \mathrm{~mm}$; ventral $(\mathrm{V}), 7.0 \mathrm{~mm}$; recording electrode in CA1: Br., $-5.6 \mathrm{~mm}$; $\mathrm{L}, 4.3 \mathrm{~mm}$; V, $1.6-3.1 \mathrm{~mm}$, respectively]. To prevent the exposed brain tissue from drying, it was covered with warm paraffin oil.

Stimulation protocols and data acquisition. Electrical stimulation of RE was performed with the use of an electrode array of three stainless steel wires (diameter, $60 \mu \mathrm{m}$, insulated except the tip). They were glued together in a glass micropipette, with tips obliquely arranged to cover the rostrocaudal extent of the (unilateral) RE. Because this nucleus is very small ( $\sim 2 \mathrm{~mm}$ in length and $\sim 0.6 \mathrm{~mm}$ in diameter) it can be easily mechanically damaged by electrode position adjustment. We therefore followed the strategy of placing the electrode array stereotaxically and fixing it in place. Stimulation was done between different pairs of the electrode array. As it turned out during the course of experiments, this strategy also allowed for a differential stimulation of either the entire rostrocaudal RE or of the rostral or caudal portion of the nucleus, separately. The standard stimulation protocol consisted of monopolar paired pulses. The first stimulus of a pair is referred to as the conditioning pulse, the second one as the test pulse. Both stimuli were of equal strength and duration [0.2 msec; interpulse interval (IPI), $100 \mathrm{msec}$, unless stated otherwise; intensity, $150-650 \mu \mathrm{A} ; 0.13 \mathrm{~Hz}$. Occasionally, trains of single pulses were applied at different frequencies ranging from $0.13-10 \mathrm{~Hz}$.

CA1 depth profiles of evoked extracellular field potentials were typically obtained using an array of six equally spaced metal electrodes (diameter, $60 \mu \mathrm{m}$, insulated except the tip; interelectrode distance, 250 $\mu \mathrm{m})$. They were arranged in the same plane, glued in a glass micropipette, and then cut at an angle of $20-30^{\circ}$. In this way we were able to record simultaneously, and along a track approximately perpendicular to the curved longitudinal axis of the hippocampus, the laminar responses across CA1, from the overlying deep cortical layers and white matter down to the hippocampal fissure (Fig. 1A,B). Evoked field potentials were amplified and digitized by way of an interface (CED 1401 plus) connected to a personal computer. They were sampled at a rate of $5000 / \mathrm{sec}$, averaged ( $n=32$, unless stated otherwise), and stored for off-line analysis.

Unit activity was recorded using a glass electrode (15-30 $\mathrm{M} \Omega$, filled with $2 \%$ Pontamine sky blue in $0.5 \mathrm{~m}$ sodium acetate buffer) that could be gradually lowered by way of a remotely controlled hydraulic manipulator. The signal was amplified and bandpass-filtered $(500-3000 \mathrm{~Hz})$. A window discriminator (WPI) was used to single out spike events, the output of which was fed to a digital port of the CED interface. Unit activity was stored as individual sweeps together with simultaneously recorded field potentials.

Off-line analysis. The characteristics of CA1 field potentials to RE stimulation were studied in laminar depth profiles. Response latencies were defined as the time from the onset of the stimulus artifact to the peak of the conditioning response. Because RE-CA1 afferents are known to form axospinous and axodendritic synapses (Wouterlood et al., 1990) (M. J. Dolleman-Van der Weel and M. P. Witter, unpublished observations), latencies corresponding to a monosynaptic RE input were to be expected. Our latencies, however, turned out to vary over a large range of values. Therefore, we additionally examined whether early (likely monosynaptic) and late (presumed disynaptic) responses showed a relationship with differences in recording or stimulation sites. Based on histological analysis, we sorted the experiments with regard to: (1) the site of recording in septal-to-temporal CA1, and (2) the site of stimulation in rostral-to-caudal RE. Subsequently, experiments were sorted according to latencies (early vs late) and then with respect to recording and stimulation sites.

The application of paired stimuli provided the opportunity to study a particular form of short term plasticity, termed paired pulse facilitation (PPF). PPF was expressed numerically by the ratio between test response amplitude/conditioning response amplitude and was calculated for elicited field potentials at the synaptic level (stratum L-M).

Whether stimulation of RE was sufficient to discharge pyramidal cells and/or interneurons was further analyzed in extracellular recordings of unit activity throughout the depth of field CA1. The latency of synaptic unit activity was measured as the time from the stimulus onset to the moment of occurrence of the spike. Simultaneously recorded field potentials were averaged to provide the corresponding population response.

Technical and theoretical considerations for current-source-density analysis. A one-dimensional current-source-density (CSD) analysis estimates the sites where currents flow into or out of the extracellular space during cellular activity (Freeman and Stone, 1969; Freeman and Nicholson, 1975). Some theoretical and practical issues, however, have to be taken into account. First, the recording should be in the plane of maximal activation, i.e., corresponding to the orientation of the apical dendrites of pyramidal cells. In this way amplitude errors of calculated currents should be minimal. A second point of importance is the distance between consecutive recording electrodes. In our experiments $100 \mu \mathrm{m}$ interval CSD profiles were recorded using a glass electrode or, alternatively, a specially constructed array of 18 metal electrodes (diameter, 60 $\mu \mathrm{m}$, insulated except the tip). These stainless steel wires were tightly glued together in the same plane and then cut at an angle of $20-30^{\circ}$ (electrode heart-to-heart distance, $100 \mu \mathrm{m}$ ). This "knife-like" electrode array, not thicker than the diameter of the wires used, caused remarkably little damage. Moreover, its shape also met the criterion for recording perpendicular to the longitudinal axis of CA1.

Histological control. At the end of the experiment, under deep anesthesia, stainless steel electrodes were marked by lesions according to a procedure (three pulses of $1 \mathrm{~mA}$ anodal current) that results in a blue (because of the potassium ferrocyanide in the fixative; see below) spot in the brain tissue, occasionally with a hole in the center of the lesion. Glass micropipettes were marked by passing current $(20 \mathrm{~min}$, electrode as cathode) to eject Pontamine sky blue. The animal was then decapitated, and the brain was quickly removed and stored for $3 \mathrm{~d}$ in $4 \%$ paraformaldehyde and $0.05 \%$ glutaraldehyde in $0.1 \mathrm{M}$ phosphate buffer with potassium ferrocyanide. The tissue was cryoprotected by immersion in $2 \%$ dimethylsulfoxide and $20 \%$ glycerin in phosphate buffer until equilibrium. On a freezing microtome the brain was cut in coronal sections (40 $\mu \mathrm{m})$ that were Nissl-stained and used for verification of electrode placements.

Neuroanatomical anterograde tracing. In the course of this study, we observed monosynaptic and presumably disynaptic responses in CA1 after RE stimulation. In a previous anatomical study, Wouterlood et al. (1990) found that injections of the anterograde tracer Phaseolus vulgaris leucoagglutinin in caudal RE resulted in dense fiber labeling in rostral RE. Their findings suggest a connection from the caudal to the rostral portion of RE that may form the anatomical substrate for our electrophysiological observations. To specifically elucidate this issue, we performed additional anatomical tracing experiments, using another five Wistar rats. The neuroanatomical tracer biotin-conjugated dextran amine (BDA; Molecular Probes, Eugene, OR) was used as described in detail elsewhere (Dolleman-Van der Weel et al., 1994). Briefly, rats were deeply anesthetized with a 4:3 parts mixture of Aescoket (1\% ketamine; Aesculaap BV, Boxtel, The Netherlands) and Rompun (2\% xylazine; Bayer, Leverkussen, Germany) and then mounted in a stereotaxic frame. At coordinates derived from those of Paxinos and Watson (1986), BDA was iontophoretically applied (pulsed positive DC current for $10 \mathrm{~min}$, 5-6.5 $\mu \mathrm{A}, 7 \mathrm{sec}$ on $/ 7 \mathrm{sec}$ off) to the caudal portion of RE. After 7-10 d of survival, the animals received an overdose of Nembutal (sodium pentobarbital; Ceva, Paris, France) and were transcardially perfused with $0.9 \%$ saline solution, followed by $4 \%$ paraformaldehyde and $0.05 \%$ glutaraldehyde in $0.1 \mathrm{~m}$ phosphate buffer, $\mathrm{pH}$ 7.4. The brain was removed from the skull, post-fixed for $1 \mathrm{hr}$, and then cryoprotected by immersion in $2 \%$ dimethylsulfoxide and $20 \%$ glycerin in phosphate buffer. On a 
A

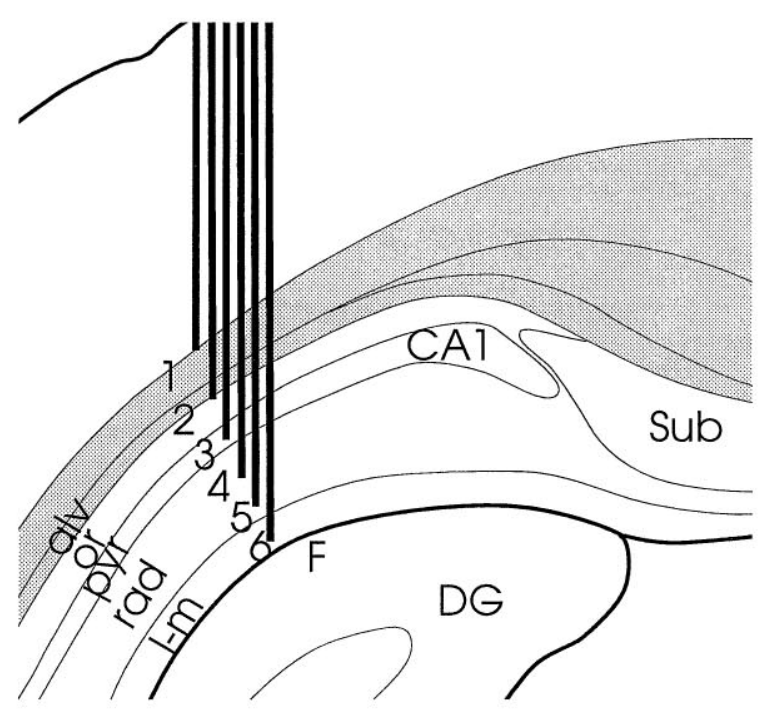

B

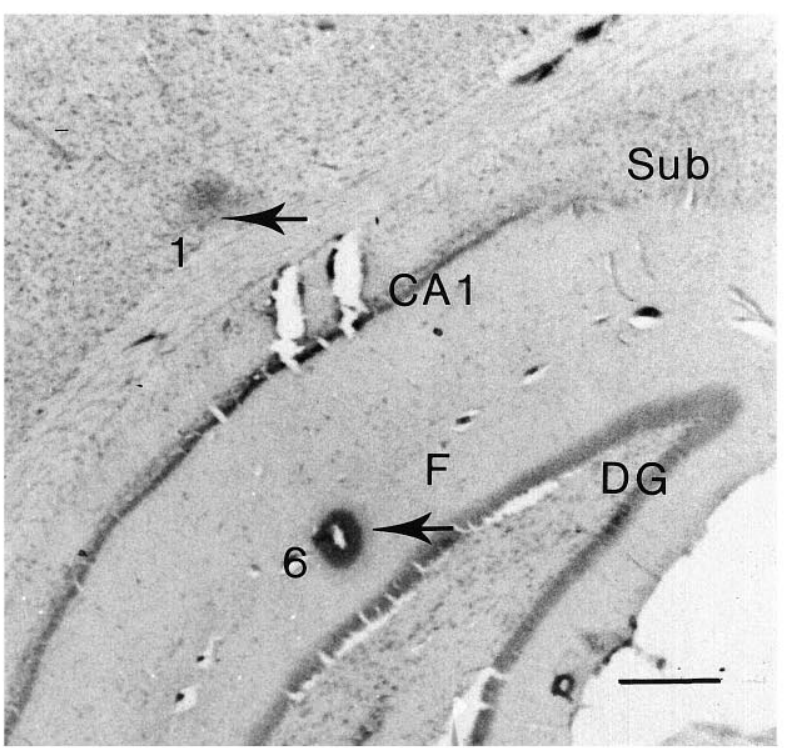

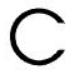

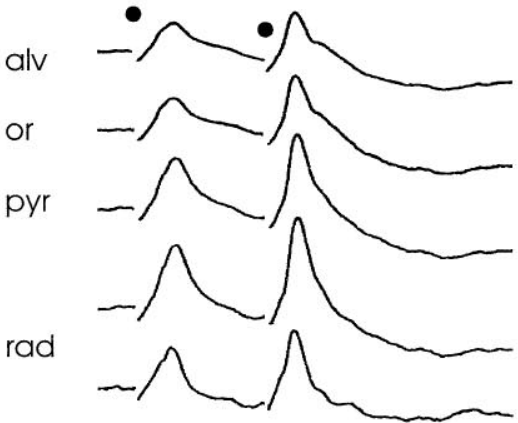

I-m
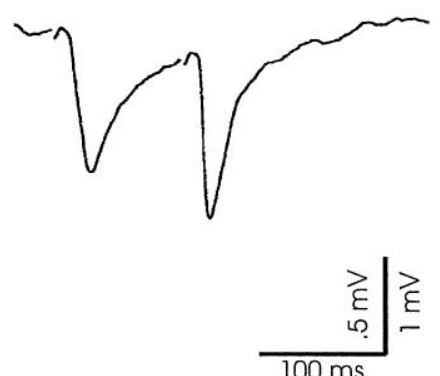

D
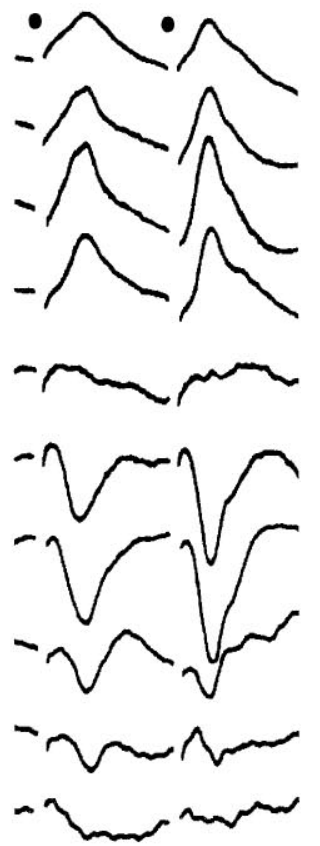

E

or

pyr

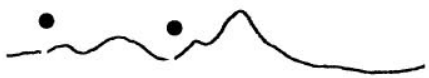

rad

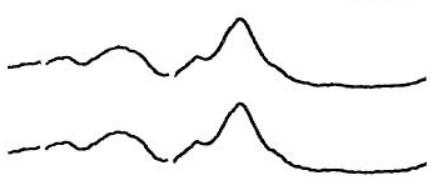

$\mathrm{rad} / \mathrm{lm}$

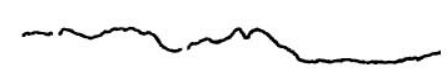

I-m

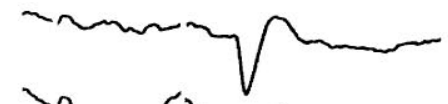

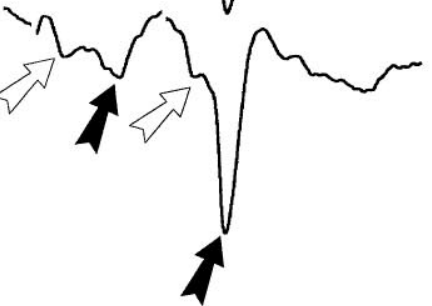

Figure 1. $A, B$, Schematic representation of a six-channel recording probe and recording site in CA1. $A$, Depth profiles of the CA1 response to RE stimulation were simultaneously recorded using a probe consisting of six equally spaced metal electrodes (interelectrode distance, $250 \mu \mathrm{m}$ ). Generally, such an array of electrodes covered a track through the depth of CA1 from the alveus (alv) down to the hippocampal fissure ( $F)$. Sub, Subiculum; $C A 1$, CA1 cell layer; or, stratum oriens; pyr, stratum pyramidale; rad, stratum radiatum; l-m, stratum lacunosum-moleculare; $D G$, dentate gyrus. $B$, Representative example of a CA1 recording site. Lesions (arrows) mark the positions of the most superficial (1) and deepest (6) electrodes in the white matter-deep cortical layer and at the fissure, respectively. Scale bar, $500 \mu \mathrm{m}$. $C-E$, Laminar CA1 field potentials to RE stimulation. $C$, Simultaneously recorded, typical CA1 profile evoked by paired pulse stimulation (high intensity stimuli; interpulse interval, $100 \mathrm{msec} ; 0.13 \mathrm{~Hz}$ ) of the rostral part of RE. The dipole field consists of a prominent negative-going deflection at the synaptic level, i.e., in the stratum lacunosum-moleculare (l-m), and a positive-going deflection in strata radiatum ( rad), pyramidale ( pyr), and oriens (or) up to the alveus (alv). The markedly increased amplitude of the second field potential illustrates the commonly induced paired pulse facilitation. Notice also the absence of a population spike. $D$, Laminar CA1 responses recorded using a glass electrode that was lowered from the alveus down to the granular cell layer in the dentate gyrus $(D G)$. The dipole field is similar to that recorded using a six-channel probe (see $C$ ). In addition, it is nicely shown that the polarity reverses at the border of strata (Figure legend continues) 
freezing microtome, serial coronal sections (40 $\mu \mathrm{m}$ thick) were cut and were immunocytochemically processed for visualization of BDA using an avidin-biotin-peroxidase complex (Vectastain ABC kit; Vector Laboratories, Burlingame, CA). Following $1.5 \mathrm{hr}$ of incubation with ABC solution, the sections were thoroughly rinsed and reacted with nickelenhanced diaminobenzidine as a chromogen. BDA-stained sections were then mounted, Nissl-stained, dehydrated, and coverslipped with Entellan (Merck, Darmstadt, Germany).

\section{RESULTS}

\section{Electrophysiological observations}

\section{$C A 1$ field response to $R E$ stimulation}

In CA1 a consistent dipole field was recorded in response to stimulation of RE in vivo. In all cases, the depth profiles, recorded with either metal or glass electrodes, were characterized by a long latency, prominent negative-going deflection in the stratum L-M that reversed polarity at the border of strata L-M and radiatum and a positive-going one in the stratum radiatum up to the alveus (Fig. $1 C-E$ ). Whenever recording electrodes were lowered into the dentate gyrus, the negative deflection of the CA1 response showed a steady decline in amplitude (Fig. 1D). This latter observation is in line with the anatomically demonstrated absence of RE projections to the dentate gyrus and field CA3 (Herkenham, 1978; Wouterlood et al., 1990; Dolleman-Van der Weel and Witter, 1996). A similar fading of the positive deflection was noticed when recordings were made in the deep cortical layers overlying CA1. Occasionally, the depth profile had a more complex waveform as documented in Figure $1 E$. In those cases, the CA1 responses consisted of two consecutive deflections (i.e., an "early," usually smaller, and a "late," larger, potential; see below) in both conditioning and test response. Yet, irrespective of waveform complexity, a common feature of the laminar depth profiles was that the largest negative peaks occurred in the stratum L-M, close to the hippocampal fissure; the largest positive peaks (as a rule smaller in amplitude than the negative ones) were recorded in the stratum radiatum. We consistently observed that a population spike could not be induced, even when the stimulus intensity was increased from 100 to $650 \mu \mathrm{A}$, and the frequency was raised from 0.13 to $10 \mathrm{~Hz}$. In fact, during stimulation at frequencies in the theta range, the peak of the field potential markedly declined, whereas the amplitude and duration of the decay phase became enhanced (data not illustrated).

\section{CSD analysis}

Laminar depth profiles (Fig. $2 A$ ) for CSDs, obtained either by the use of a probe with 18 metal electrodes (electrode heart-to-heart distance, $100 \mu \mathrm{m}$ ) or by stepwise lowering of a glass electrode (100 $\mu \mathrm{m}$ interval) from the white matter down to the hippocampal fissure, revealed a well defined sink at the level of the stratum L-M and a clear source in the stratum radiatum (Fig. $2 B$ ). The source declined in amplitude toward the strata pyramidale and oriens. This sink-source configuration is compatible with the interpretation that RE input in the stratum L-M elicits a field EPSP (fEPSP) in the apical dendrites of pyramidal cells.

\section{Paired pulse facilitation}

The short term dynamic properties of the RE-CA1 projection were studied by analyzing evoked fEPSPs to double pulse stimulation of RE (fixed IPI, $100 \mathrm{msec} ; 0.13 \mathrm{~Hz}$ ) at different stimulus intensities. PPF was quantified by the ratio between test/conditioning peak and calculated for responses recorded in the stratum L-M, representing the summed active RE-CA1 synaptic processes.

In general, the amplitude of the conditioning deflection was quite small at low (150-300 $\mu \mathrm{A})$ and moderate $(350-450 \mu \mathrm{A})$ intensities but was better distinguishable at high stimulus intensity $(500-650 \mu \mathrm{A})$. RE-induced PPF of fEPSPs was robust at low to high intensity stimuli (low intensity: mean PPF, $1.5 \pm 0.2 ; n=$ 7; moderate intensity: mean PPF, $2.1 \pm 0.8 ; n=6$; high intensity: mean PPF, $1.9 \pm 0.8 ; n=18)$. We also examined PPF resulting from paired stimuli (high intensity, $0.13 \mathrm{~Hz}$ ) at IPIs ranging from 20 to $200 \mathrm{msec}$. At $200 \mathrm{msec}$ IPI, PPF was comparable to that elicited by stimuli at $100 \mathrm{msec}$ IPI; at IPIs shorter than $100 \mathrm{msec}$, PPF was as robust as at $100 \mathrm{msec}$ IPI or displayed a slight increase in magnitude. Paired pulse depression of the test deflection under the stimulus conditions used was not observed.

\section{Synaptic unit activity to RE stimulation}

The consistent absence of a population spike in our CA1 profiles indicated the probable paucity of pyramidal cell discharge to RE stimulation. Careful examination of all depth profiles revealed that stimulus-triggered spike events did occur, but only in two experiments. In both cases, however, the spikes were not detected at the pyramidal cell level; rather, they were superimposed on the fEPSPs recorded in the distal stratum radiatum in both conditioning and test response (Fig. 3A). Thus the radiatum spikes can be considered synaptically elicited in radiatum interneurons. The latencies of the radiatum spikes in these two experiments were remarkably similar (21 and $22 \mathrm{msec}$, respectively) and shorter than the latency of the fEPSP peak. In addition, we noticed that radiatum spikes were generated only after high intensity stimulation of RE and at low frequencies (range, 0.13-2 Hz; see Fig. $3 B$ ); they disappeared when we applied stimuli at frequencies in the theta range $(5-10 \mathrm{~Hz})$ but reappeared when stimulation was resumed at low rates (e.g., at $0.13 \mathrm{~Hz}$ ).

Unit activity was systematically investigated in 11 cases by stepwise lowering of a glass electrode across CA1 from the alveus down to the hippocampal fissure. Low to high intensity paired stimuli (IPI, $100 \mathrm{msec} ; 0.13 \mathrm{~Hz}$ ) were applied to RE, and unit activity and fEPSPs were recorded simultaneously. In general, many spontaneously active neurons were encountered approaching the pyramidal cell layer, but they became more sparse when the electrode was lowered through strata radiatum and L-M. Only one synaptically driven neuron was recorded at the oriens/alveus border. This neuron generated a short latency $(9 \mathrm{msec})$ action potential to both the conditioning and test pulses (Fig. 3C) at low as well as high intensity stimulation of RE. The simultaneously recorded, small amplitude deflection at the oriens/alveus level (Fig. 3D) shows that the RE-elicited oriens/alveus spike preceded the local field response.

radiatum/lacunosum-moleculare $(\mathrm{rad} / \mathrm{l}-\mathrm{m})$. When the electrode was lowered through the fissure $(\mathrm{fis})$ into the dentate gyrus $(D G)$, the negative deflection rapidly declined toward the granular cell level (bottom trace). E, Example of an occasionally recorded complex CA1 response, resulting from stimulation of the caudal part of RE. This CA1 dipole field displays a configuration similar to that of the profiles shown in $C$ and $D$. However, early (open arrow) as well as late (black arrow) potentials are present in both conditioning and test response. The early field potentials in these complex responses are usually of small amplitude. Stimulation moments in $C-E$ are indicated by dots. 
A

CA1 depth profile

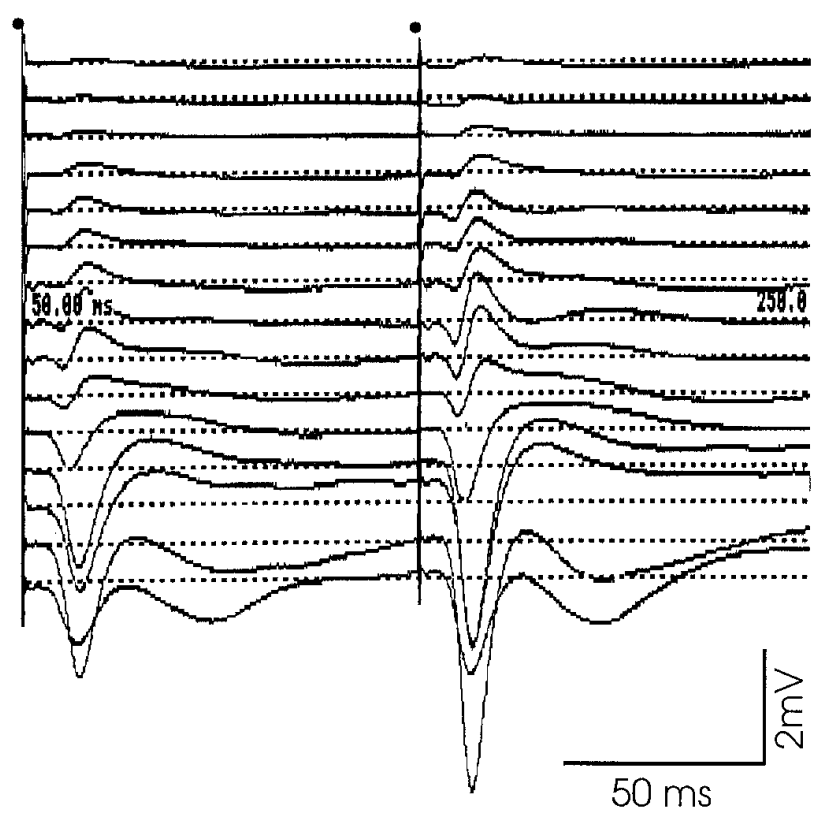

B

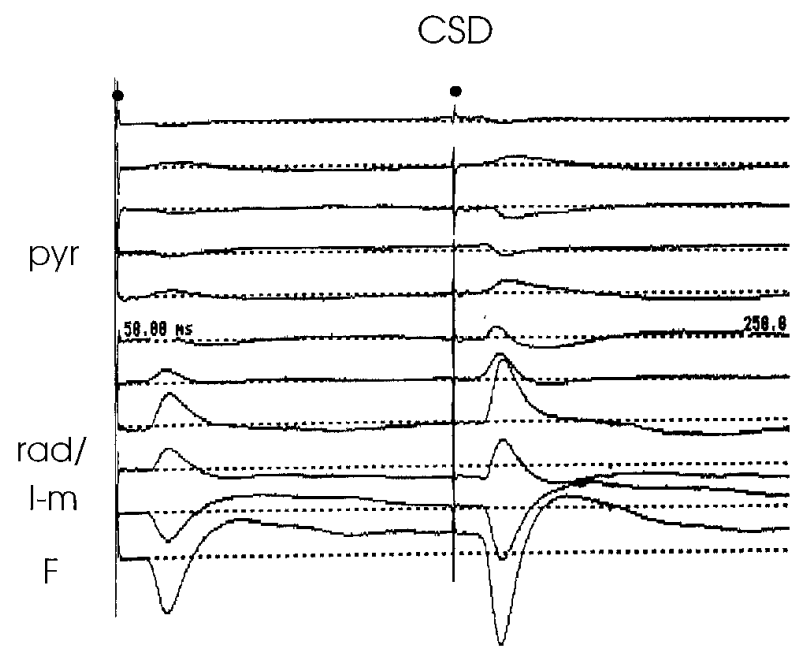

Figure 2. Depth profile of RE-evoked field potentials in CA1 $(A)$ with the corresponding CSD $(B)$. $A$, The depth profile was recorded using a probe with 18 metal electrodes (see Materials and Methods; electrode heart-to-heart distance, $100 \mu \mathrm{m})$. Dots indicate the moments of the paired stimuli (100 msec interpulse interval, $0.13 \mathrm{~Hz}$ ). The recordings from electrodes $4-18$ are depicted, covering CA1 from the white matter overlying the hippocampus down to just across the hippocampal fissure into the dentate gyrus. Indicated are the pyramidal cell layer ( $p y r$ ), the radiatum/lacunosum-moleculare border $(\mathrm{rad} / \mathrm{l}-\mathrm{m})$, and the fissure $(F)$. Note that in the bottom trace, recorded just below the fissure, the negative deflection is much smaller compared with the trace recorded above the fissure (also see Fig. $1 D$ ). $B$, The CSD, corresponding to the depth profile shown in $A$, clearly demonstrates the prominent lacunosum-moleculare sink; the radiatum source rapidly declines toward the pyramidal cell layer (sinks are shown by a deflection downward, sources by a deflection upward, in arbitrary units).

\section{Monosynaptic and disynaptic nature of RE-evoked fEPSPS in $C A 1$}

Because RE afferents in CA1 form axospinous and axodendritic contacts (Wouterlood et al., 1990), the RE input was thus expected to be of a monosynaptic nature. During the series of experiments, however, we found a rather wide range (13-39 msec) of latencies of fEPSPs to RE stimulation. Therefore, we analyzed whether these responses were monosynaptically and/or disynaptically evoked, possibly in relation to differences in recording or stimulation sites. Based on histological verification of electrode placements, the experiments were sorted for recording site (septal-to-temporal CA1) and stimulation site (rostral-tocaudal RE), respectively. By subsequent comparison with the sorting for early versus late responses, we found that so-called early (monosynaptic) responses (mean latency, $16.8 \pm 3.6 \mathrm{msec}$; $n=18$ ) were evoked by stimulation of the rostral two-thirds of RE (rRE; Fig. 4A,C); late (presumably disynaptic) responses (mean latency, $33.0 \pm 3.2 \mathrm{msec} ; n=6$ ) resulted from stimulation of caudal RE (cRE; Fig. 4A,D). Occasionally we recorded complex responses $(n=5)$, such as the one documented in Figure $1 E$, displaying early as well as late deflections in both conditioning and test response. In those complex field potentials the early conditioning and test fEPSPs were usually of small amplitude (see Fig. $1 E$ ). Because the (subtle) differences in CA1 recording sites could not be associated with any variation in response latency or waveform complexity, we concluded that the source of the disynaptic nature of RE-CA1 input must be within RE itself.

\section{Anatomical observations}

Intranucleus projection from caudal to rostral $R E$

We reasoned that an intranucleus connectivity between caudal and rostral RE might underlie the observed monosynaptic and disynaptic nature of rostral or caudal RE-evoked fEPSPs in CA1. Therefore, we investigated whether a projection that connects the caudal and rostral parts of the nucleus could be demonstrated. The neuroanatomical tracer BDA was injected in the caudal RE, where it was incorporated by neurons confined to the caudal portion of the nucleus (Fig. $5 A$ ). BDA is primarily transported in an anterograde direction. Uptake of BDA by (damaged) fibers of passage may result in some retrograde transport (Veenman et al., 1992); anterograde transport of BDA in axons of passage has not been reported. Our BDA injections resulted in many anterogradely labeled varicose fibers and terminal labeling in rostral RE (Fig. 5B,C). In comparison with the dense terminal labeling in rostral RE resulting from injections in caudal RE, we detected a sparse terminal labeling in hippocampal field CA1. This latter observation confirms previous findings (Wouterlood et al., 1990; Dolleman-Van der Weel and Witter, 1996) that caudal RE gives rise to only a minor innervation of CA1. When BDA was injected 


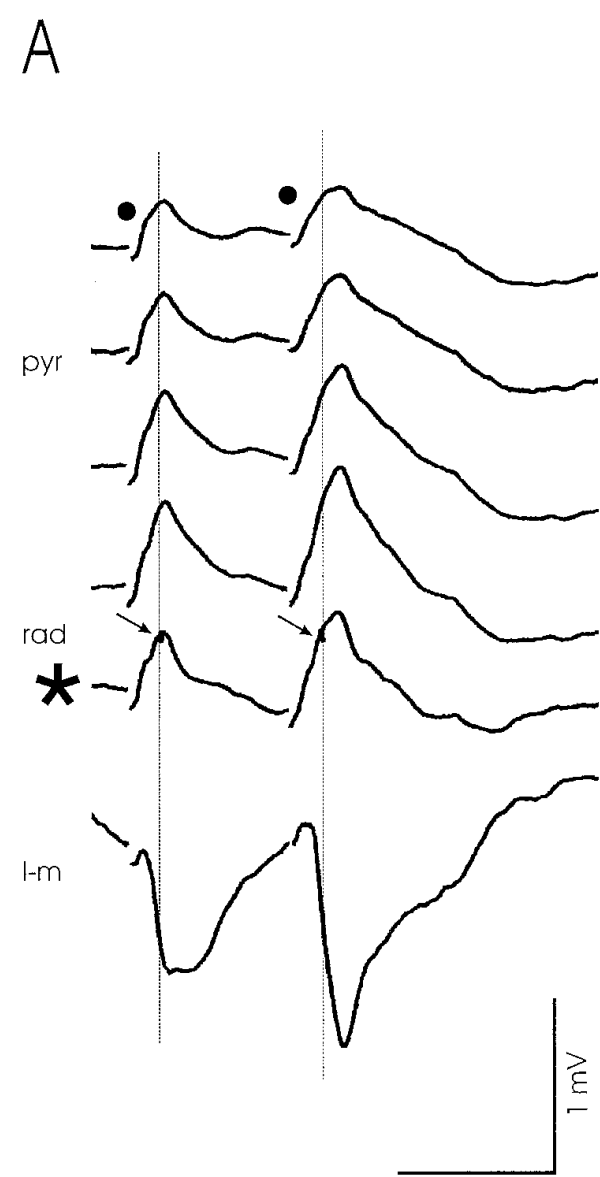

$100 \mathrm{~ms}$
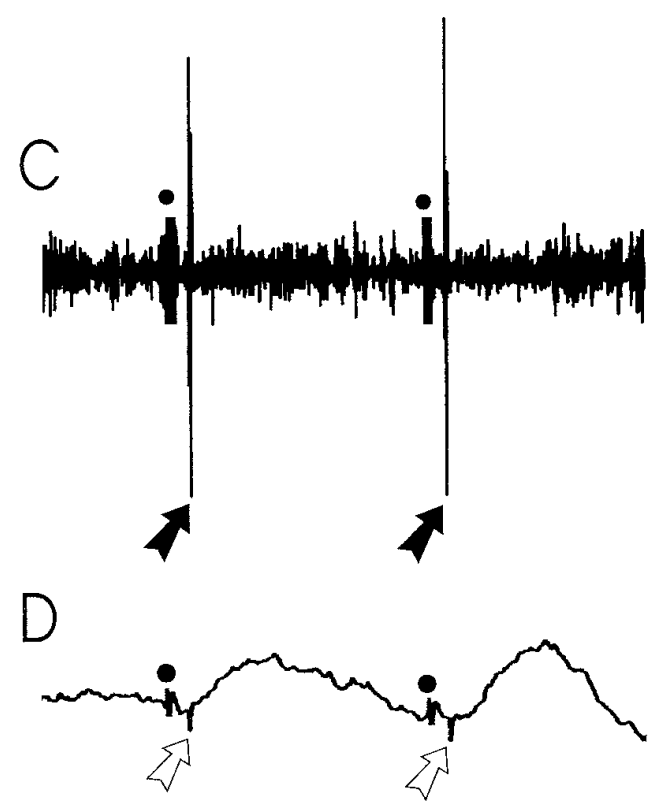

$50 \mathrm{~ms}$
B

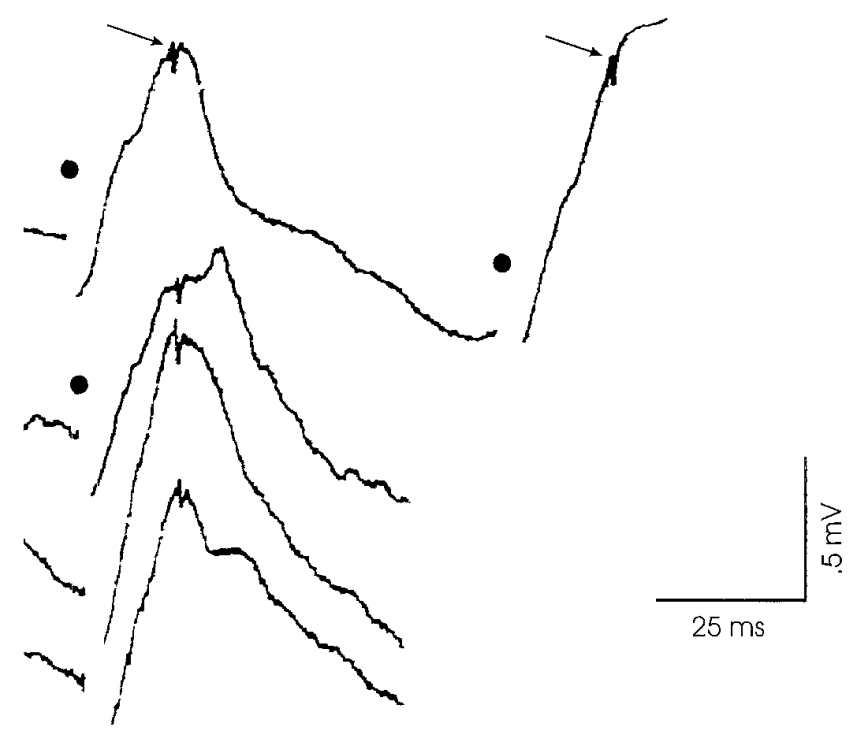

C
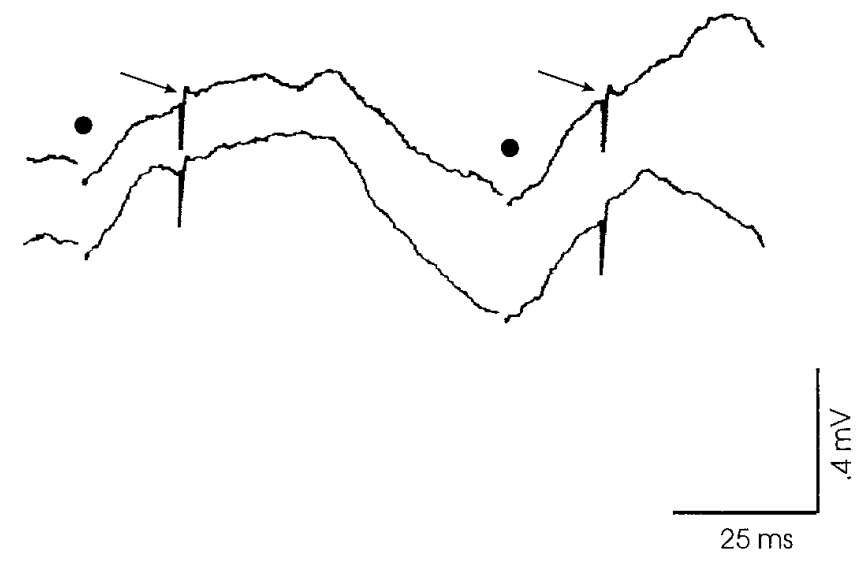

Figure 3. RE-evoked spikes in the distal stratum radiatum and at the oriens/ alveus border, indicative of the activation of local interneurons. A, A simultaneously recorded CA1 depth profile reveals that stimulus-triggered spikes (arrows) occurred only in the distal stratum radiatum $\left(\mathrm{rad},{ }^{*}\right)$, in both the conditioning and test response, after high intensity stimulation of RE ( 0.13 Hz). pyr, stratum pyramidale; $l-m$, stratum lacunosum-moleculare; $B$, Magnification of recordings from the stratum radiatum. $a$, Radiatum spikes (arrows) shown in $A$ (trace marked ${ }^{*}$ ). $b$, Radiatum field potentials to single pulses applied to RE at frequencies in the range from 0.13 to $2 \mathrm{~Hz}$ also exhibited spikes. $c$, Stimulus-triggered radiatum spikes (arrows) in both conditioning and test response, recorded in another rat. Notice that in all cases the latencies of the radiatum spikes were highly comparable. $C$, A synaptically driven neuron was encountered at the oriens/alveus border. A short latency action potential (black arrows) was recorded in both the conditioning and test response. $D$, The simultaneously recorded local field potential shows that the RE-evoked oriens/ alveus spike (open arrows) preceded the postsynaptic pyramidal cell response. Stimulation moments in $A-D$ are indicated by dots. 
A
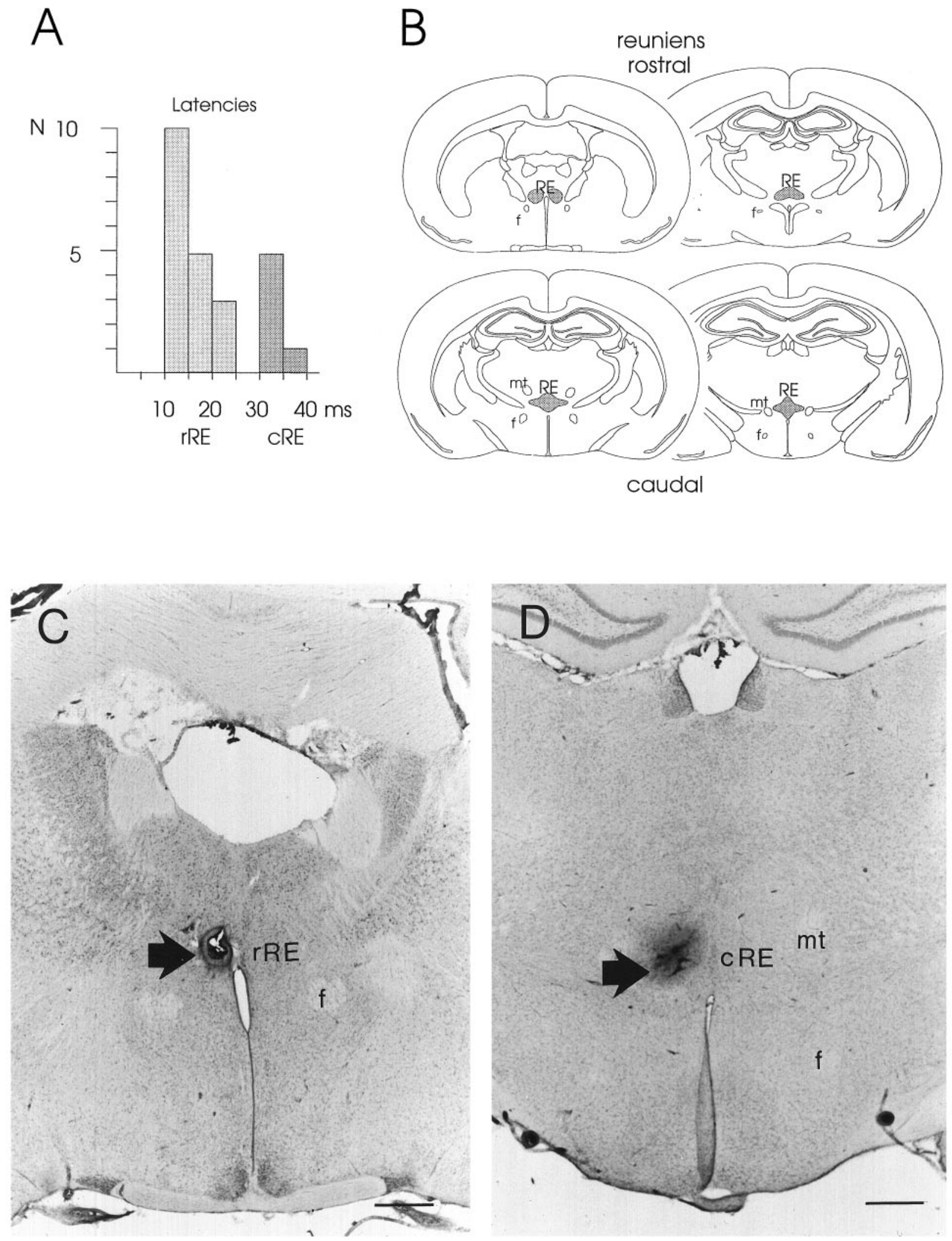

Figure 4. Stimulation of rostral versus caudal RE and associated early versus late CA1 responses. $A$, The histogram represents the distribution of latencies of CA1 field potentials corresponding with early or late responses to stimulation of rRE or cRE, respectively. $B$, Series of rostral-to-caudal coronal sections through the rat brain, illustrating the location of the rostral (top row) and caudal (bottom row) portions of RE (shaded areas). $C$, Representative example of the position of a stimulation electrode in rostral RE, marked by a small lesion (arrow). $D$, Representative example of a stimulation site in caudal RE. The dark spot, representing the center of the lesion (arrow), indicates the position of the stimulation electrode. $f$, Fornix; $m t$, mammillothalamic tract. Scale bars, $500 \mu \mathrm{m}$. 

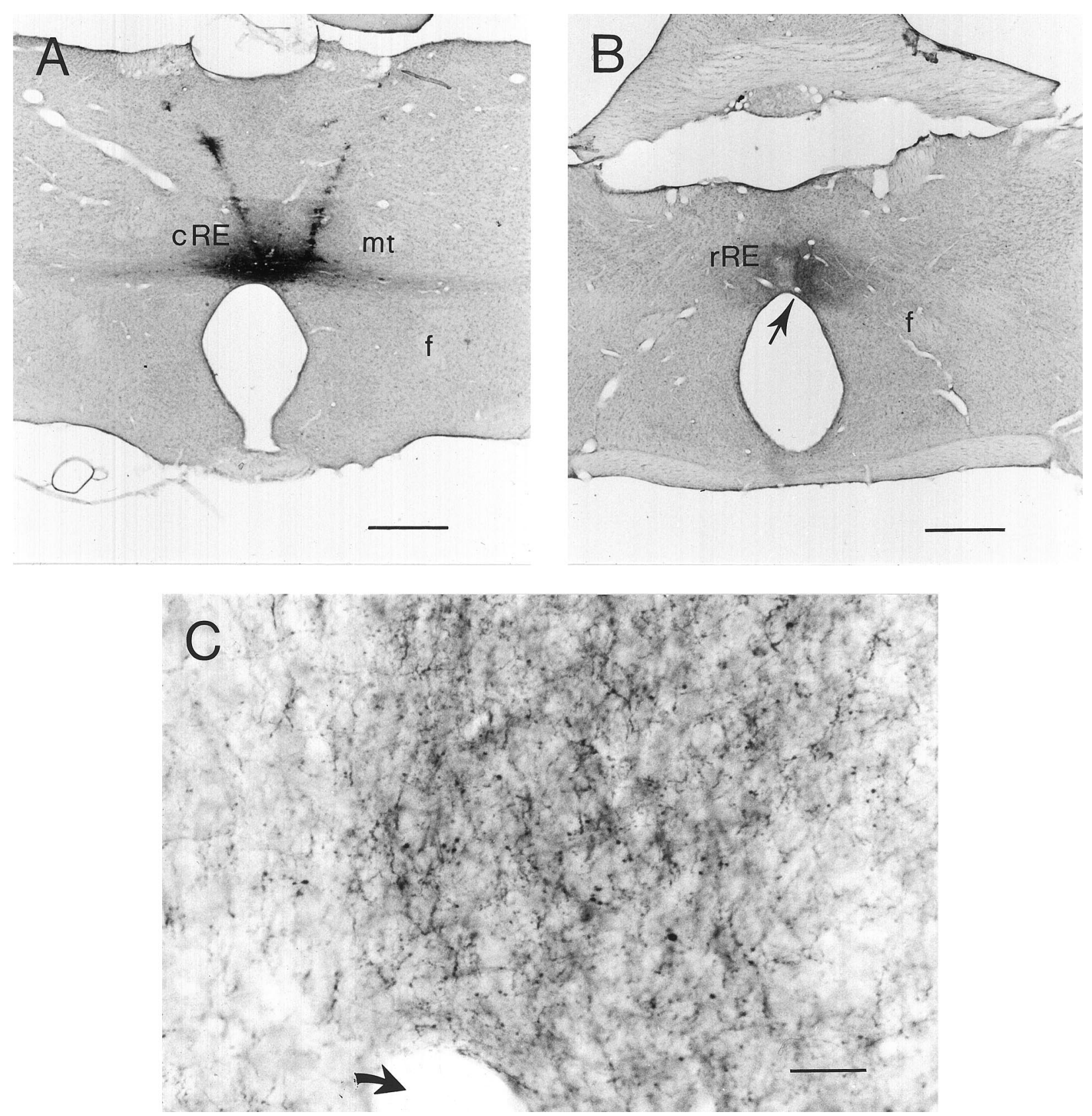

Figure 5. Intranucleus projection from caudal to rostral RE. $A$, Representative example of an injection site in caudal RE; the anterograde tracer BDA has been incorporated by neurons located in the caudal part of the nucleus. $B$, The injection shown in $A$ resulted in a dense plexus of BDA-positive varicose fibers and terminal labeling in the rostral part of RE. $C$, High magnification of the terminal labeling in rostral RE shown in $B$. Arrows in $B$ and $C$ indicate the same blood vessel. Scale bars: $A$ and $B, 500 \mu \mathrm{m} ; C, 20 \mu \mathrm{m}$. $f$, Fornix; $m t$, mammillothalamic tract.

in the poorly delineated thalamic area just caudal to RE, labeled fibers were absent in both rostral RE and field CA1.

\section{DISCUSSION}

The present study provides the first electrophysiological evidence (schematically summarized in Fig. 6) that RE is able to modulate transmission in CA1 through both excitatory and inhibitory mechanisms. First, electrical stimulation of RECA1 afferents at their origin causes an active (subthreshold) depolarization of the apical dendrites of pyramidal cells, which may enhance their state of excitability. Second, RE-elicited spiking of extracellularly recorded units was not detected in the stratum pyramidale but in strata oriens/alveus and radia- tum, which indicates synaptic excitation of local nonpyramidal cells that are likely associated with inhibitory mechanisms (Lacaille et al., 1987; Samulack et al., 1993; McBain et al., 1994; Steffensen, 1995; Bergles et al., 1996; Maccaferri and McBain, 1996; for review, see Freund and Buzsáki, 1996). Furthermore, evidence is provided for the existence of a projection from caudal to rostral RE that is considered to form the anatomical substrate underlying the presently observed disynaptic and complex RE-evoked responses in CA1. Because caudal RE receives input from the hippocampus via the subiculum (Herkenham, 1978; Witter and Groenewegen, 1990; Dolleman-Van der Weel et al., 1993), we propose that the caudal to rostral RE connection might act to close a novel 


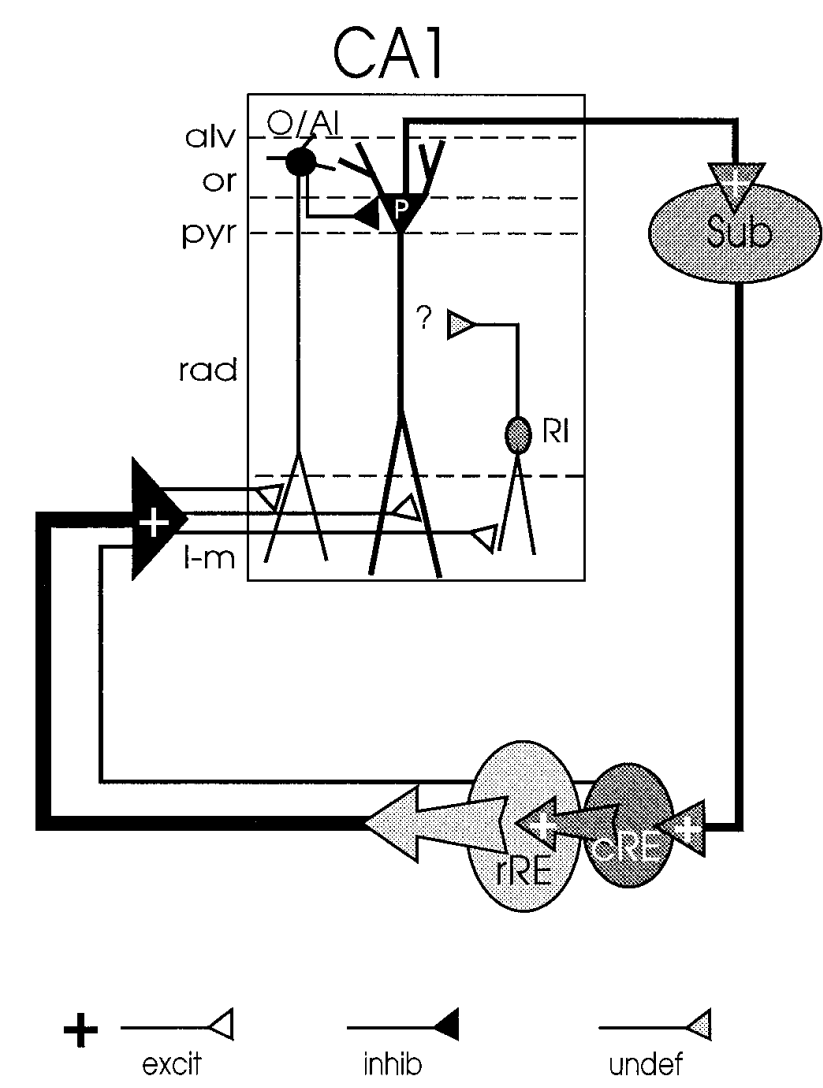

Figure 6. Schematic representation of the RE-CA1-subiculum-RE loop. The RE-CA1 input is both monosynaptically and disynaptically organized. Monosynaptic input originates predominantly in the rRE; only a minor portion of the monosynaptic afferents arises from $\mathrm{cRE}$. A dense intranucleus projection from cRE to rRE can account for the disynaptic cRE-rRE-CA1 input. In the stratum lacunosum-moleculare $(l-m)$ of CA1, the RE axons form exclusively asymmetrical [i.e., excitatory (excit)] synapses on the apical dendrites of pyramidal cells $[P$; stratum pyramidale $(p y r)]$, as well as on those of subtypes of interneurons located at the oriens $(o r) /$ alveus $(a l v)$ border [vertical oriens/alveus interneuron $(O / A I)]$ and in the distal stratum radiatum $[\mathrm{rad}$; radiatum interneuron $(R I)]$. Electrical stimulation of RE in vivo elicits a subthreshold depolarization in pyramidal cells and the generation of synaptic spikes in oriens/alveus and radiatum interneurons. The vertical oriens/alveus interneurons are assumed to mediate feedforward inhibition (inhib) of pyramidal cells; the axonal targets of radiatum interneurons (the latter probably containing both inhibitory and excitatory transmitters [undefined (undef); see Discussion], and thereby the role these interneurons play in the local circuit of CA1 awaits further investigation. Major output of CA1 is known to be transmitted to the subiculum (Sub), which, in turn, projects back to cRE. This suggests a closed rRE-CA1-subiculum-cRE-rRE loop that may enable RE to modulate the flow of information through CA1 depending on the output of the hippocampus.

circuit (see Fig. 6) between rostral RE-CA1-subiculum-caudal RE-rostral RE, which allows RE to modulate the activity level in CA1, depending on the output of the hippocampus.

The L-M sink-radiatum source configuration is in agreement with the interpretation that stimulation of RE evokes a synaptic EPSP in the apical dendrites of pyramidal cells. However, this distal EPSP seems insufficient to elicit action potentials in these cells. This can be attributable to its spatial decay in the proximal direction, likely associated with an inhibitory action at the somatic level (see compartmental-volume-conduction model by Leung, 1995). The latter is in line with the observation that RE is able to discharge interneurons that are likely inhibitory and are known to contact the pyramidal cell bodies (see below). In CSDs, however, synaptically elicited activity by nonpyramidal cells can remain undetected, because these cells: (1) are widely distributed and largely outnumbered by CA1 cells, and (2) display a laminar dendritic orientation similar to that of pyramidal cells.

The RE-evoked dipole field, lacking a population spike, is remarkably similar to that evoked by stimulation of the excitatory entorhinal cortex (EC)-CA1 input in the rat (Colbert and Levy, 1992; Empson and Heinemann, 1995; Leung, 1995; Levy et al., 1995). Thus, like EC-evoked CA1 responses, RE-evoked fEPSPs may be mediated by non-NMDA as well as NMDA receptors (Colbert and Levy, 1992; Empson and Heinemann, 1995). However, the pharmacology of CA1 responses to RE stimulation awaits further investigation. The presently observed conspicuous RE-induced PPF of fEPSPs, noted to be largely independent on stimulus intensity and IPI duration (at least under all tested conditions), indicates that RE can exert a persistent influence on the state of pyramidal cell excitability. This will probably keep the latter cells close to the firing threshold, allowing them to discharge under certain conditions, for instance, during periods of diminished inhibition. Previously, the findings by Hirayasu and Wada (1992a,b) also indirectly pointed to the ability of RE to modulate temporal limbic excitability. These investigators observed that NMDA injections in RE caused remarkable behavioral and temporal lobe EEG changes, i.e., tonic and/or clonic generalized convulsions, and seizure discharges.

Our interpretation that RE is able to elicit a suprathreshold activation of interneurons is based on the detection of elicited spikes in the stratum radiatum and at the oriens/alveus border. Direct evidence for this interpretation (i.e., RE-evoked responses in morphologically identified interneurons) should be obtained in future studies using the in vivo intracellular recording and labeling technique (e.g., Sik et al., 1995). Nevertheless, the present assumption is consistent with the observations that: (1) stimulation of RE never elicited action potentials in the stratum pyramidale; (2) stimulus-triggered action potentials were found in strata containing interneurons that, from a morphological view point (see below), can be contacted by RE fibers; and (3) anatomically we found that RE-CA1 axons form asymmetrical synapses on GABA-positive dendrites in the stratum L-M (M. J. DollemanVan der Weel and M. P. Witter, unpublished results). These latter observations support our interpretation that RE-elicited spiking of extracellularly recorded units reflects monosynaptic activation of interneurons. With respect to the observed radiatum spike events, however, we also have to address the possibility that these actually represented dendritic spiking in pyramidal cells. High intensity, low frequency stimulation of radiatum fibers and Schaffer collaterals (Herreras, 1990) has been shown to result in the consistent occurrence of dendritic spikes generated by CA1 pyramidal cells. In our hands, using a standard protocol of high intensity stimulation at $0.13 \mathrm{~Hz}$, dendritic spiking thus should have been encountered on a far more regular basis than the two cases in which triggered spikes in the stratum radiatum were detected. Moreover, the latency of the radiatum spikes in those two experiments was highly comparable, despite a difference in latency between the respective fEPSPs. This supports the interpretation that RE-elicited radiatum spikes reflect synaptic discharges in radiatum interneurons.

According to their distinctive morphologies, axonal targets, and neurochemical markers, CA1 interneurons in strata oriens/ alveus and radiatum represent a heterogeneous class of nonpyramidal cells (McBain et al., 1994; Buckmaster and Soltesz, 1996; Freund and Buzsáki, 1996; Maccaferri and McBain, 1996). A 
prerequisite for these interneurons to receive $\mathrm{RE}$ input is that their dendritic tree extends into the stratum L-M. This has been shown for a subpopulation of cells at the oriens/alveus border, the so-called vertical oriens/alveus cells (McBain et al., 1994). These interneurons have an extensively arborizing axon that is largely confined to the stratum pyramidale, forming symmetrical (inhibitory) synapses on the somata and primary dendrites of numerous CA1 cells. Functionally, they are assumed to mediate both feedforward and feedback inhibition of pyramidal cells (Lacaille et al., 1987; Lacaille and Schwarzkroin, 1988; Samulack et al., 1993). Interneurons located in the distal radiatum that fulfill the criterion to receive RE input are likely the ones containing GABA as well as the putative excitatory transmitters cholecystokinin (CCK) and/or vasoactive intestinal polypeptide (VIP) (Kosaka et al., 1985; Gulyás et al., 1991; Haas and Gáhwiler, 1992; Acsády et al., 1996a,b). GABA-CCK cells have been observed to form symmetrical synapses with pyramidal cell bodies (Harris et al., 1985; Nunzi et al., 1985); GABA-VIP cells have been shown to contact primarily other interneurons (Acsády et al., 1996a,b; Freund and Buzsáki, 1996). Although the function of these interneuron subtypes in the stratum radiatum remains to be established, based on their different axonal targets it can be assumed that each subtype plays a distinctive role in the local circuit of CA1. Thus, the ability to discharge interneuron subpopulations in strata oriens/alveus and radiatum (e.g., differing in discharge threshold, afferent and efferent targets, and transmitter contents) increases the flexibility of RE to modulate transmission in CA1. The possible interaction between the EC-CA1 and RE-CA1 inputs is also of interest in understanding the role RE might play in modulating the activity in the entorhinal-hippocampal circuit. In this respect, our preliminary results from paired stimulation of $\mathrm{RE}$ and EC revealed that an interaction (i.e., facilitation of elicited-fEPSPs, yet without inducing a population spike) between perforant path and RE inputs occurs in CA1 (M. J. Dolleman-Van der Weel and F. H. Lopez da Silva, unpublished results).

Based on the previously demonstrated RE-CA1 (asymmetrical) synapses on spines and dendrites (Wouterlood et al., 1990), a monosynaptic RE input was to be expected. However, RE-evoked CA1 responses displayed a wide range of latency values, indicating that both monosynaptic and disynaptic RE inputs exist. Interestingly, our early (monosynaptic) and late (disynaptic) CA1 responses seemed associated with selective stimulation of rostral and caudal RE, respectively. Anatomically, rostral RE is the major source of CA1 afferents, whereas caudal RE contributes very modestly to this projection (Dolleman-Van der Weel and Witter, 1996). We now report that caudal RE gives rise to a dense innervation of rostral RE. Taken together, our anatomical and electrophysiological data thus suggest that the caudal-to-rostral $\mathrm{RE}$ projection is involved in the disynaptic, or occasionally noted complex monosynaptic and disynaptic, fEPSPs in CA1 evoked by stimulation of caudal RE. In these latter complex responses, the early potential of a small amplitude likely represents the activation of few caudal RE neurons projecting monosynaptically to CA1; the late potential of a larger amplitude then reflects the activation of the presently described caudal RE-rostral RE-CA1 disynaptic input. Because caudal RE receives input from the hippocampus via the subiculum (Herkenham, 1978; Witter and Groenewegen, 1990; Dolleman-Van der Weel et al., 1993), we propose the existence of a closed circuit (see Fig. 6) between rostral RE-CA1-subiculum-caudal RE-rostral RE, which may allow RE to modulate the activity level in CA1 depending on the hippocampal output.

\section{REFERENCES}

Acsády L, Arabadzisz D, Freund TF (1996a) Correlated morphological and neurochemical features identify different subsets of vasoactive intestinal polypeptide-immunoreactive interneurons in rat hippocampus. Neuroscience 73:299-315.

Acsády L, Görcs TJ, Freund TF (1996b) Different populations of vasoactive intestinal polypeptide-immunoreactive interneurons are specialized to control pyramidal cells or interneurons in the hippocampus. Neuroscience 73:317-334.

Bergles DE, Doze VA, Madison DV, Smith SJ (1996) Excitatory actions of norepinephrine on multiple classes of hippocampal CA1 interneurons. J Neurosci 16:572-585.

Braak H, Braak E (1991) Alzheimer's disease affects limbic nuclei of the thalamus. Acta Neuropathol (Berl) 81:261-268.

Braak H, Braak E (1992) The human entorhinal cortex: normal morphology and lamina-specific pathology in various diseases. Neurosci Res 15:6-31.

Buckmaster PS, Soltesz I (1996) Neurobiology of hippocampal interneurons: a workshop review. Hippocampus 6:330-339.

Colbert CM, Levy WB (1992) Electrophysiological and pharmacological characterization of perforant path synapses in CA1: mediation by glutamate receptors. J Physiol (Lond) 68:1-8.

Dolleman-Van der Weel MJ, Witter MP (1996) Projections from the nucleus reuniens thalami to the entorhinal cortex, hippocampal field CA1, and the subiculum in the rat arise from different populations of neurons. J Comp Neurol 364:637-650.

Dolleman-Van der Weel MJ, Ang W, Witter MP (1993) Afferent connections of the nucleus reuniens thalami: a neuroanatomical tracing study in the rat. Eur J Neurosci [Suppl] 6:247.

Dolleman-Van der Weel MJ, Wouterlood FG, Witter MP (1994) Multiple anterograde tracing, combining Phaseolus vulgaris leucoagglutinin with rhodamine- and biotin-conjugated dextran amine. J Neurosci Methods 51:9-21.

Empson RM, Heinemann U (1995) The perforant path projection to hippocampal area CA1 in the rat hippocampal-entorhinal cortex combined slice. J Physiol (Lond) 484:707-720.

Freeman JA, Nicholson C (1975) Experimental optimization of currentsource-density technique for anuram cerebellum. J Neurophysiol 38:369-382.

Freeman JA, Stone JA (1969) A technique for current source density analysis of field potentials and its application to the frog cerebellum. In: Neurobiology of cerebellar evolution and development (Llinás R, ed), pp 421-430. Chicago: American Medical Association.

Freund TF, Buzsáki G (1996) Interneurons of the hippocampus. Hippocampus $6: 347-470$.

Gulyás AI, Tóth K, Dános P, Freund TF (1991) Subpopulations of GABAergic neurons containing parvalbumin, calbindin D28k, and cholecystokinin in the rat hippocampus. J Comp Neurol 312:371-378.

Haas HL, Gáhwiler BH (1992) Vasoactive intestinal polypeptide modulates neuronal excitability in hippocampal slices of the rat. Neuroscience 47:273-277.

Harris KM, Marshall PE, Landis DM (1985) Ultrastructural study of cholecystokinin-immunoreactive cells and processes in area CA1 of the rat hippocampus. J Comp Neurol 233:147-158.

Herkenham M (1978) The connections of the nucleus reuniens thalami: Evidence for a direct thalamo-hippocampal pathway in the rat. J Comp Neurol 177:589-609.

Herreras O (1990) Propagating dendritic action potential mediates synaptic transmission in CA1 pyramidal cells in situ. J Neurophysiol 64:1429-1441.

Hirayasu Y, Wada JA (1992a) $N$-methyl-D-aspartate injection into the massa intermedia facilitates development of limbic kindling in rats. Epilepsia 33:965-970.

Hirayasu Y, Wada JA (1992b) Convulsive seizures in rats induced by $N$-methyl-D-aspartate injection into the massa intermedia. Brain Res 577:36-40.

Kosaka T, Kosaka K, Tateishi K, Hamaoka Y, Yanaihara N, Wu J-Y, Hama K (1985) GABAergic neurons containing CCK-8-like and/or VIP-like immunoreactivities in the rat hippocampus and dentate gyrus. J Comp Neurol 239:420-430. 
Lacaille J-C, Schwarzkroin PA (1988) Stratum lacunosum-moleculare interneurons of hippocampal CA1 region. II. Intrasomatic and intradendritic recordings of local circuit synaptic interactions. J Neurosci $8: 1411-1424$.

Lacaille J-C, Mueller AL, Kunkel DD, Schwarzkroin PA (1987) Local circuit interactions between oriens/alveus interneurons and CA1 pyramidal cells in hippocampal slices: electrophysiology and morphology. J Neurosci 7:1979-1993.

Leung L-WS (1995) Simulation of perforant path evoked field and intracellular potentials in hippocampal CA1 area. Hippocampus 5:129-136.

Levy WB, Colbert CM, Desmond NL (1995) Another network model bites the dust: entorhinal inputs are no more than weakly excitatory in the hippocampal CA1 region. Hippocampus 5:137-140.

Maccaferri GM, McBain CJ (1996) Long-term potentiation in distinct subtypes of hippocampal nonpyramidal neurons. J Neurosci 16:5334-5343.

McBain CJ, DiChiara TJ, Kauer JA (1994) Activation of metabotropic glutamate receptors differentially affects two classes of hippocampal interneurons and potentiates excitatory synaptic transmission. J Neurophysiol 14:4433-4445.

Nunzi MG, Gorio A, Milan F, Freund TF, Somogyi P, Smith AD (1985) Cholecystokinin-immunoreactive cells form symmetrical synaptic contacts with pyramidal and non-pyramidal neurons in the hippocampus. J Comp Neurol 237:485-505.

Paxinos G, Watson C (1986) The rat brain in stereotaxic coordinates, Ed 2. New York: Academic.

Rousseau M (1994) Amnesias following limited thalamic lesions. In: The memory system of the brain, advanced series in neuroscience Vol 4 (Delacour J, ed), pp 241-277. Singapore: World Scientific.

Samulack DD, Williams S, Lacaille J-C (1993) Hyperpolarizing synaptic potentials evoked in CA1 pyramidal cells by glutamate stimulation of interneurons from the oriens/alveus border of rat hippocampal slices. I. Electrophysiological response properties. Hippocampus 3:331-344.

Sik A, Penttonen M, Ylinen A, Buzsáki G (1995) Hippocampal CA1 interneurons: an in vivo intracellular labeling study. J Neurosci 15:6651-6665.

Steffensen SC (1995) Dehydroepiandrosterone sulfate suppresses hippocampal recurrent inhibition and synchronizes neuronal activity to theta rhythm. Hippocampus 5:320-328.

Squire LR (1992) Memory and the hippocampus: a synthesis from findings with rats, monkeys and humans. Physiol Rev 99:195-231.

Vanderwolf CH, Leung L-WS, Cooley RK (1985) Pathways through cingulate, neo- and entorhinal cortices mediate atropine-resistant hippocampal rhythmical slow activity. Brain Res 347:58-73.

Veenman CL, Reiner A, Honig MG (1992) Biotinylated-dextran amine as an anterograde tracer for single- and double labelings studies. J Neurosci Methods 41:239-254.

Witter MP, Groenewegen HJ (1990) The subiculum: cytarchitectonically a simple structure, but hodologically complex. Prog Brain Res 83:47-58.

Wouterlood FG, Saldana E, Witter MP (1990) Projection from the nucleus reuniens thalami to the hippocampal region: light and electron microscopic tracing study in the rat with the anterograde tracer Phaseolus vulgaris leucoagglutinin. J Comp Neurol 296:179-203. 